\title{
Toward richer metadata for microbial sequences: replacing strain-level NCBI taxonomy taxids with BioProject, BioSample and Assembly records
}

\author{
Scott Federhen ${ }^{1}$, Karen Clark' ${ }^{1}$ Tanya Barrett ${ }^{1}$, Helen Parkinson ${ }^{2}$, James Ostell ${ }^{1}$, Yuichi Ko- \\ dama $^{3}$, Jun Mashima ${ }^{3}$, Yasukazu Nakamura ${ }^{3}$, Guy Cochrane ${ }^{2}$, and Ilene Karsch-Mizrachi ${ }^{1}$. \\ ${ }^{1}$ National Center for Biotechnology Information, National Library of Medicine, National In- \\ stitutes of Health, Bethesda, MD, USA \\ ${ }^{2}$ European Molecular Biology Laboratory, European Bioinformatics Institute, Wellcome \\ Trust Genome Campus, Hinxton, UK \\ ${ }^{3}$ DDBJ Center, National Institute of Genetics, Research Organization for Information and \\ Systems, Yata, Mishima, Japan
}

Microbial genome sequence submissions to the International Nucleotide Sequence Database Collaboration (INSDC) have been annotated with organism names that include the strain identifier. Each of these strain-level names has been assigned a unique 'taxid' in the NCBI Taxonomy Database. With the significant growth in genome sequencing, it is not possible to continue with the curation of strain-level taxids. In January 2014, NCBI will cease assigning strain-level taxids. Instead, submitters are encouraged provide strain information and rich metadata with their submission to the sequence database, BioProject and BioSample.

\section{Toward richer metadata for microbial sequences}

The NCBI taxonomy database provides the organism nomenclature and classification that is used in sequence entries by the International Nucleotide Sequence Database Collaboration (INSDC [1]; comprising GenBank, ENA and the DDBJ) [2]. The NCBI Taxonomy Group is responsible for curating names for taxa that are regulated by the relevant codes of nomenclature [3-5], for providing informal names for specimens that are not identified with Linnaean species binomials, and for maintaining the 'taxid' namespace. This is a laborintensive and largely manual effort undertaken by this small group of diligent and dedicated taxonomists at the NCBI [6].

It has been almost twenty years since the first bacterial genomes started to appear in the sequence databases, beginning with Haemophilus influenzae in 1995, followed within a year by Escherichia coli. In those days each new genome sequence was of significant scientific interest and represented a considerable technical achievement. At that time, for the convenience of those at INSDC institutes and their users, the taxonomy group started assigning strain-level taxids for prokaryotes with complete genome sequences, e.g.: "Haemophilus influenzae Rd" [7] and "Escherichia coli K12" [8].
(That genome is currently indexed as "Escherichia coli str. K-12 substr. MG1655", since there are now many genomes sequenced from 'strain' K-12.) Since that time, the policy of assigning strain-level taxids for genome sequences has been extended to cover eukaryotic microbes as well - unicellular fungi, algae and protists - but it has never been applied to the multicellular eukaryotes. In particular, strain-level taxids have never been assigned for breeds of dogs, or for inbred strains of mice, or for individual human genomes.

Sequencing technology has undergone remarkable development over the past twenty years and it has become increasingly cheap and easy to sequence genomes, a trend that promises to continue in the foreseeable future. We are already seeing the submission of hundreds of genomes at a time that are simply time points of micro-evolutionary studies in Escherichia coli, or Saccharomyces cerevisiae. Another growing industry in genome submissions is in efforts to track epidemics, food-borne illnesses and hospital infection pathways. More will appear as this technology finds applications in other fields.

Our recognition that the curation of strain-level taxids will not remain possible under such growth, and that alternative data resources relating to biological samples are maturing at the INSDC partner institutes, has led us to a review of our practices in 
this area. We intend to discontinue the curation of strain-level taxids for microbial genomes submitted beyond January 2014. Importantly, this change in practice will not be applied retrospectively; we will not remove any of the thousands of strainlevel nodes that we have added in the past, and we will continue to add informal strain-specific names for genomes from specimens that have not been identified to the species level, e.g.: "Rhizobium sp. CCGE 510" and "Salpingoeca sp. ATCC 50818".

We strongly encourage submitters to annotate their genome submissions with the relevant source metadata, including strain, culture collection and isolation information as appropriate, plus the appropriate species (or subspecies) name. The Genomic Standards Consortium maintains checklists of Minimal Information about any (x) Sequence (MIxS) [9] that contain mandatory and optional descriptive metadata fields for a variety of organism types. These MIxS checklists can be included in the genome submission.

Our alternative system for recording and presenting strain-level annotation will be provided by the respective BioSample databases of the INSDC partner institutes [10-12]. BioSample records provide a single accessioned unit of information relating to a sample that has been assayed using sequencing or other platforms. This information serves to gather together taxonomic information, informal infraspecies information (such as strain), descriptors relating to the sampling process, accession information for the physical sample itself, etc.

For genome submissions, INSDC databases guide submitters through a series of logical steps in which the information required is requested and transferred. An early step is the registration of the initiative (BioProject) or indication that the genome data are connected to an existing initiative (This registration is applied within the INSDC host institutes' respective BioProject and study databases). Following this, users are prompted to provide rich descriptive information about the sequenced sample(s) (BioSample) or an indication that samples already registered have been sequenced. Description of new samples, and updates and enhancements to existing samples, take advantage of defined checklists or 'packages' of attributes, appropriate for the initiative. In later steps of the genome submission process, users provide sequence data and functional annotation that connect to the samples described or selected.

BioSample records are one tool that can be used as an organizing and retrieval key to the genome datasets, as the strain-level taxid was in the past. BioSample accessions can be used to aggregate submitted data deposited in various archives, such as those that cover sequence (i.e. INSDC) and those that cover array-based studies (such as GEO, ArrayExpress and the DDBJ Omics Archive) [1214]. The BioSample record will enable users to retrieve data across databases from samples with particular attributes. For instance, one may wish to retrieve submitted data for all Salmonella enterica strains isolated from a particular agricultural plant.

INSDC assembly records are another powerful tool in this area, as these hold the information about a particular genome assembly and are supported with unique assembly-level identifiers. In these records all of the pieces of a genome are collected together in ways that are much more flexible and powerful for indexing and retrieval purposes than were strain-level taxids. For example, genomes representing independent assemblies of the same sequence data share a BioSample accession, while those representing alternative sequencing studies of the same strain may have independent BioSample accessions.

The Streptococcus pneumoniae TIGR4 (taxid 170187) genome initiative is described in PRJNA766132. This record contains two genome assembles that were built from sequence reads from a single BioSample, SAMN001035273. Two different assembly algorithms were used to create the assemblies, which are detailed in GCA_000269665 ${ }^{4}$ and GCA_0002734455.

In an era when microbial genome sequencing was not as commonplace as it is now, using a taxid as a key to retrieve the genome and associated project metadata was a reasonable approach. However, with next-generation sequencing technology, one can sequence the genomes of hundreds of closely related microbes in a few hours [15]. Therefore, data consumers are better served by the new resources that we describe above that enable them to retrieve sets of genomes based on common attributes or initiatives.

The INSDC is prepared to stop assigning strainlevel tax ids for strains of microbes that have their genome sequenced by January 2014 and encourages users to exploit other resources that allow 
them to explore sequence data by initiative, specimen or genome assembly.

\section{Acknowledgments}

This work was supported in part by the Intramural Research Program of the NIH, National Library of Medicine, the European Molecular Biology Laboratory, and the Ministry of Education, Culture, Sports, Science and Technology of Japan (MEXT).

\section{References}

1. INSDC. http://www.insdc.org/

2. Nakamura Y, Cochrane G, Karsch-Mizrachi I. The International Nucleotide Sequence Database Collaboration. Nucleic Acids Res 2013; 41(D1):D21D24. PubMed http://dx.doi.org/10.1093/nar/gks1084

3. Ride WDL, Cogger HG, Dupuis C, Kraus O, Minelli A, Thompson FC, Tubbs PK, eds. International Code of Zoological Nomenclature. 1999. 4th edn. International Trust for Zoological Nomenclature, The Natural History Museum, London http://www.nhm.ac.uk/hostedsites/iczn/code/

4. McNeill J, Barrie FR, Burdet HM, Demoulin V, Hawksworth DL, Marhold K, Nicolson DH, Prado J, Silva PC, Skog JE, et al. International Code of Botanical Nomenclature (Vienna Code). Regnum Veg 2006; 146

5. LaPage SP, Sneath PHA, Lessel EF, Skerman VBD, Seeliger HPR, Clark WA, eds. International Code of Nomenclature of Bacteria: Bacteriological Code (1990 Revision) 1992. ASM Press, Washington D.C.

http://www.ncbi.nlm.nih.gov/books/NBK8817/

6. Federhen S. The NCBI Taxonomy database. Nucleic Acids Res 2012; 40(D1):D136-D143. PubMed http://dx.doi.org/10.1093/nar/gkr1178

7. Fleischmann RD, Adams MD, White O, Clayton RA, Kirkness EF, Kerlavage AR, Bult CJ, Tomb JF, Dougherty BA, Merrick JM, et al. Whole-genome random sequencing and assembly of Haemophilus influenza Rd. Science 1995;

269:496-512. PubMed http://dx.doi.org/10.1126/science.7542800

8. Blattner FR, Plunkett G, III, Bloch CA, Perna NT, Burland V, Riley M, Collado-Vides J, Glasner JD, Rode CK, Mayhew GF, et al. The complete genome sequence of Escherichia coli K-12. Science
1997; 277:1453-1462. PubMed

http://dx.doi.org/10.1126/science.277.5331.1453

9. Yilmaz P, Kottmann R, Field D, Knight R, Cole JR, Amaral-Zettler L, Gilbert JA, Karsch-Mizrachi I, Johnston A, Cochrane G, et al. Minimum information about a marker gene sequence

(MIMARKS) and minimum information about any (x) sequence (MIxS) specifications. Nat Biotechnol 2011; 29:415-420. PubMed

http://dx.doi.org/10.1038/nbt.1823

10. Barrett T, Clark K, Gevorgyan R, Gorelenkov V, Gribov E, Karsch-Mizrachi I, Kimelman M, Pruitt

K, Resenchuk S, Tatusova T, et al. BioProject and BioSample databases at $\mathrm{NCBI}$ : facilitating capture and organization of metadata. Nucleic Acids Res 2012; 40(D1):D57-D63. PubMed http://dx.doi.org/10.1093/nar/gkr1163

11. Gostev M, Faulconbridge A, Brandizi M, Fernandez-Banet J, Sarkans U, Brazma A, Parkinson H. The BioSample Database (BioSD) at the European Bioinformatics Institute. Nucleic Acids Res 2012; 40(D1):D64-D70. PubMed http://dx.doi.org/10.1093/nar/gkr937

12. Kodama Y, Mashima J, Kaminuma E, Gojobori T, Ogasawara O, Takagi T, Okubo K, Nakamura Y. The DNA Data Bank of Japan launches a new resource, the DDBJ Omics Archive of functional genomics experiments. Nucleic Acids Res 2012; 40(D1):D38-D42. PubMed http://dx.doi.org/10.1093/nar/gkr994

13. Barrett T, Wilhite SE, Ledoux P, Kim IF, Tomashevsky M, Marshall KA, Phillippy KH, Sherman PM, Holko M, Yefanov A, et al. NCBI GEO: archive for functional genomics data setsupdate. Nucleic Acids Res 2013; 41(D1):D991D995. PubMed http://dx.doi.org/10.1093/nar/gks1193

14. Rustici G, Kolesnikov N, Brandizi M, Burdett $T$, Dylag M, Emam I, Farne A, Hastings E, Ison J, Keays $M$, et al. ArrayExpress update-trends in database growth and links to data analysis tools. Nucleic Acids Res 2013; 41:D987-D990. PubMed http://dx.doi.org/10.1093/nar/gks1174

15. Hu B, Xie G, Lo CC, Starkenburg SR, Chain PSG. Pathogen comparative genomics in the nextgeneration sequencing era: genome alignments, pangenomics and metagenomics. Briefings in Functional Genomics. 2011; 10:322-333. PubMed http://dx.doi.org/10.1093/bfgp/elr042 